\title{
Effects of Stereopsis and Head Tracking on Performance Using Desktop Virtual Environment Displays
}

\begin{abstract}
A bstract
This study investigated performance in a desktop virtual environment as a function of stereopsis and head tracking. Ten subjects traced a computer-generated wire using a virtual stylus that was slaved to the position of a real-world stylus tracked with a 6-D O F position sensor. The objective of the task was to keep the virtual stylus centered on the wire. Measures collected as the subjects performed the task were performance time, and number of times the stylus overstepped the virtual wire. The time to complete the wire-tracing task was significantly reduced by the addition of stereopsis, but was not affected by the presence of head tracking. The number of times the virtual stylus overstepped the wire was significantly reduced when head-tracking cues were available, but was not affected by the presence of stereoscopic cues. Implications of the results for performance using desktop virtual environments are discussed.
\end{abstract}

\section{Introduction}

This paper focuses on determining whether the visual cues of stereopsis and motion parallax (provided by head tracking) affect task performance when using a desktop virtual environment. In order to support training and performance in virtual environments, it is essential to provide the necessary sensory cues (e.g., auditory, spatial, or haptic) and display hardware that are required for the task. Some visual cues in virtual environments include linear perspective, binocular disparity, and motion parallax, while some hardware options include the type of display (for example, H M D , rear projection, or desktop). $N$ ote the logical relationship between display features and the availability of visual cues: the use of head tracking allows the observer to experience motion parallax, while the use of a stereoscopic display allows the observer to view stereoscopic images.

Presence, Vol. 8, N 0. 2, April 1999, 237-240 (C) 1999 by the Massachusetts Institute of Technology
$M$ any studies have investigated the effect of adding stereopsis to a perspective display. For example, Yeh and Silverstein (1992) found that the addition of stereopsis led to faster and more-accurate spatial judgments than a perspective display alone. Furthermore, Kim, Ellis, Tyler, $\mathrm{H}$ annaford, and Stark (1987) examined the effect of stereopsis and perspective cues on subjects' ability to manually track an object. Their results indicated that stereopsis improved the overall tracking performance.

Another feature of desktop virtual environments is head tracking. The addition of head tracking to a stereoscopic display provides the viewer with motion parallax, which can be used to determine relative depth relations among objects (Kaiser \& Proffitt, 1989; M cKenna \& Zeltzer, 1992). Ware, Arthur, and Booth (1993) investigated the ability of observers to perceive arterial branching in brain-scan data as a function of head tracking and stereopsis. Their results indicated that the addition of head tracking and stereopsis reduced error rates by a factor of 16 over a static pictorial display, and by a factor of 10 over a static stereoscopic display. In a related study using a similar experimental task, Rekimoto (1995) found that a stereoscopic display with head tracking resulted in longer times to perform the task compared to a stereoscopic display without head tracking. H owever, the head-tracked display resulted in a higher level of accuracy compared to performance using a stereoscopic display without head tracking. Rekimoto attributed the longer response times found for the head-tracked display to the fact that, in this condition, the movement time of the head was factored into the overall performance time.

\footnotetext{
W oodrow Barfield

Claudia Hendrix Karl-Erik Bystrom barfield@vt.edu Virginia Polytechnic Institute and State U niversity University of Minnesota U niversity of $\mathrm{W}$ ashington
} 


\section{Method}

The study was run using a $2 \times 2$ within-subjects design; there were two independent variables each at two levels: head tracking (presence or absence) and stereopsis (presence or absence). Ten subjects (nine men and one woman, with a mean age of 22.8 years) repeated each of four conditions three times. The first repetition of each condition was considered practice and was not included in the statistical analysis. The following performance measures were recorded during each trial: (1) the time to completely trace the bent wire from left to right, measured in seconds, and (2) the number of times the stylus ring overstepped the wire during a trial (termed a hit).

The experimental task was a wire-tracing task, in which the objective was to move a virtual stylus (controlled by a real stylus) along the path of a virtual wire as quickly as possible without touching the wire. The tracing task models fine motor skills required to perform actions such as those required in surgical procedures, guidance, and manual tracking. The on-screen ring was controlled by the position of a magnetically tracked physical stylus held in the subject's dominant hand. U nlike in a physical wire-tracing task, the virtual ring was not physically restricted from a transverse movement completely off the wire path, but it did indicate such a movement with a color change. The virtual ring was light yellow when on the path, and gray when it had touched or overstepped the wire path. The shape and length of the wire path was constant across all trials and all subjects.

The wire display was shown on a Silicon G raphics workstation with a screen resolution of $1280 \times 1024$ pixels on a 19-in. color monitor. H ead tracking and stylus position sensing were performed with Polhemus trackers. Stereo conditions were presented at a $120 \mathrm{H} \mathrm{z}$ refresh rate ( $60 \mathrm{H} \mathrm{z}$ for each eye), presenting an effective resolution of $1280 \times 512$ pixels. Stylus position was determined with six degrees of freedom (D O Fs), head tracking was performed with three D O Fs, (translations in $x, y$, and $z$ ). Stereoscopic conditions were created using $C$ rystalEyes time multiplexed LCD shutter glasses. The wire was projected out of the screen toward the viewer, so all disparity cues were negative or crossed and ranged from 0.30 to 0.45 degrees $(2.93 \mathrm{~mm}$ to $4.27 \mathrm{~mm}$ separation). M onoscopic conditions were viewed at a $120 \mathrm{H} \mathrm{z}$ refresh rate $(60 \mathrm{H} \mathrm{z}$ for each eye) but with the stereo disparity set to zero. With the subjects seated approximately $55 \mathrm{~cm}$ from the screen, the wire subtended approximately 35 degrees of visual angle horizontally as viewed on the screen. The physical stylus was a $15.24 \mathrm{~cm}$ length of $1.91 \mathrm{~cm}$-diameter dowel rod with a Polhemus sensor mounted on the end. The on-screen movements of the virtual stylus were yoked to the translations and rotations of the real stylus.

\section{$3 \quad$ Results}

The results of a repeated-measures AN O VA indicated that the addition of stereopsis to the display significantly reduced the mean time to complete the wire tracing task $(F(1,9)=11.07, p<0.01)$ (mean time approximately $15 \mathrm{sec}$. faster). Furthermore, the AN O VA procedure indicated that the addition of head tracking to the display did not significantly affect the time to complete the task, although performance when head tracking was implemented as a display feature was approximately $1 \mathrm{sec}$. faster than for the nonhead-tracked display $(F(1,9)=0.40, p>0.05)$. H owever, the interaction between display type and head tracking was found to be statistically significant $(F(1,9)=9.96, p<0.01)$. Figure 1 illustrates that the mean time to complete the wire-tracing task was faster when using the nonheadtracked stereoscopic display than when using either of the two monoscopic conditions (head tracked or nonhead tracked), or the head-tracked stereoscopic display condition. The combination of features that produced the slowest performance was the nonhead-tracked monoscopic display, which represents the current desktop computing environment.

The results of the AN O VA procedure indicated that the addition of head tracking to the display significantly decreased the number of hits when performing the wiretracing task $(F(1,9)=23.91, p<0.0009)$ (mean result: 17.0 hits with $\mathrm{HT}$ versus 23.1 hits without $\mathrm{HT}$ ). $\mathrm{M}$ oreover, the addition of stereopsis to the display re- 


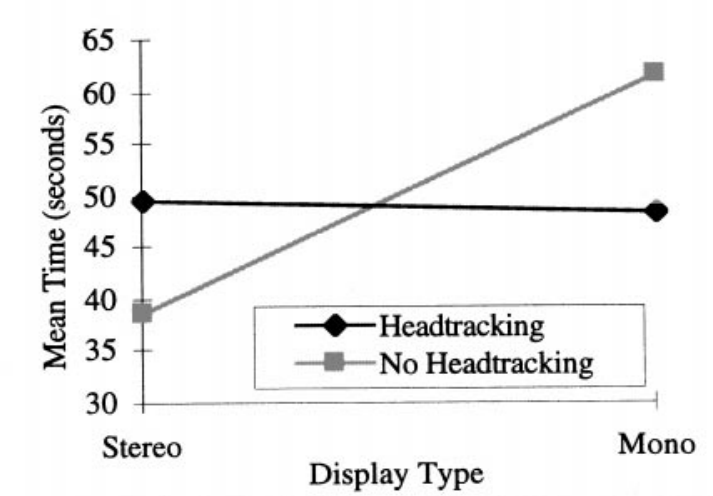

Figure 1. The two-way interaction between presence or absence of head tracking and display type for time to perform the tracing task.

sulted in a trend towards statistical significance ( $F(1$, $9)=4.25, p<0.07)$, indicating that fewer hits were produced when using the stereoscopic desktop virtual environment (18.6 versus 21.5 ). The interaction between display type and head tracking was not found to be statistically significant $(F(1,9)=2.41, p>0.05)$.

\section{Discussion}

In terms of task accuracy, the findings were largely as expected. The use of head tracking significantly improved the tracing accuracy, and the use of stereopsis had a tendency to improve the tracing accuracy. Given a desktop display, the results suggest that motion parallax cues may be of greater benefit in sparse visual scenes than binocular disparity cues. The finding that head tracking led to increased accuracy for the spatial task is consistent with the findings of Ware et al. (1993).

Somewhat puzzling are the findings on task performance time. As expected, the use of stereopsis led to faster performance time when head tracking was not implemented. H owever, the nature of the interaction between stereopsis and head tracking was unexpected. When head tracking was absent, performance time was faster with stereopsis present than with a monoscopic display; this result matched expectations. But when head tracking was present, the task performance time was al- most identical for both the monoscopic and stereoscopic conditions. When a monoscopic display with head tracking was used, performance time improved, as expected. Furthermore, when a stereoscopic display with head tracking was used, performance time was worse than with no head tracking. This finding was contrary to expectations; it was expected that the head-tracked, stereoscopic condition would lead to the fastest performance time rather than to an intermediate value. It should be noted, though, that this condition did still have the highest tracing accuracy.

Several explanations might account for this unexpected finding for the stereoscopic condition with head tracking. $O$ ne possibility is that the presence of motion parallax cues may, in some way, have interfered with the binocular disparity cues present in the stereoscopic scene. This explanation, though, does not explain why performance was more accurate when both stereopsis and head tracking were present than when only stereopsis was present. If motion parallax cues interfered with binocular disparity cues, one would expect both performance time and accuracy to be degraded. Another possible explanation for the slower wire-tracing times resulting from the stereoscopic head tracked case relates to the type of position sensor used to track the user's head. A Polhemus tracker is known to introduce lag or delays in visual feedback as the head is moved; the magnitude of the lag is influenced by data and update rates ( $M$ eyer, Applewhite, \& Biocca, 1992). H owever, although we did not measure the magnitude of the lag associated with the current experimental task, the visual scene was very sparse in terms of polygons, and the workstation was able to maintain an update rate well above $15 \mathrm{~Hz}$, producing the illusion of smooth motion as the subject moved the virtual stylus along the virtual wire. Furthermore, the subjects traced the wire slowly enough that lag and frame rate would likely have had little effect on performance; the mean times to trace the wire in the head-tracked monoscopic and stereoscopic conditions were respectively 48.3 and $49.5 \mathrm{sec}$. A third possible explanation for the slower wire-tracing times associated with the stereoscopic head-tracked condition is that allowing subjects to initiate head movements in the head- 
tracked cases may simply have led them to take more time to perform the experimental task than they would have taken otherwise. This was the case in a study by Rekimoto (1995), who found that the additional time required to initiate head movements within head-tracked stereoscopic desktop virtual environments led to increased overall performance time compared to a nonhead-tracked stereoscopic display.

These findings raise several interesting questions to be addressed in future studies. For example, is head tracking the optimum procedure to implement motion parallax, or would motion parallax cues provided by any input method sustain performance? I $f$ the mere existence of motion parallax is the salient design feature to support performance, one could easily implement motion parallax through mouse or wand tracking or some other method rather than by using head tracking. If, however, the user's mapping of head motion to the motion parallax cues is important, one would need to implement motion parallax using head tracking rather than some other method. This would be the case if an egocentric frame of reference in combination with motion parallax cues were necessary to support performance.

\section{A cknowledgments}

This work was supported in part by the Air F orce $O$ ffice of Scientific Research (Contract 92-N L-225 and I N ST PRO P NO : 78216), the $\mathrm{N}$ ational Science Foundation (D M C -8857851, CDA-8806866), and by the Center for Research in L earning, Perception, and Cognition, and the $\mathrm{N}$ ational Science Founda- tion's Program in Visual Perception and M otor Control (GER 9454163). We thank Koh Wei and J eff Brandt for their assistance with the study.

\section{References}

Kaiser, M . K., \& Proffitt, D. R. (1989). Perceptual issues in scientific visualization, three-dimensional visualization and display technologies. SPIE, I maging Sci. Technol ., 18-20.

Kim, W. S., Ellis, S. R., Tyler, M., H annaford, B., \& Stark, L. (1987). A quantitative evaluation of perspective and stereoscopic displays in three-axis manual tracking tasks. I EEE Transactions on Systems, M an, and C ybernetics, SM C - 17, 61-71.

M cKenna, M . D ., \& Zeltzer, D . (1992). Three dimensional visual display systems for virtual environments. Presence: Teleoperators and Virtual Environments, 1(4), 421-458.

M eyer, K., Applewhite, H . L., \& Biocca, F. A. (1992). A survey of position sensors. Presence: Teleoperatorsand Virtual Environments, 1 (2), 173-200.

Pepper, R. L., \& Cole, R. E. (1978). D isplay systems variables affecting operator performance in undersea vehicles and work systems. N O SC TR 268. N aval O cean Systems C enter, San Diego, CA.

Rekimoto, J . (1995). A vision-based head tracker for fish tank virtual reality-VR without head gear. Virtual R eality A nnual I nternational Symposium '95, 94-100.

Ware, C., Arthur, K., \& Booth, K. S. (1993). Fish tank virtual reality. I N TER CH I, 37-42.

Yeh, Y., \& Silverstein, L. D. (1992). Spatial judgments with monoscopic and stereoscopic presentation of perspective displays. H uman Factors, 34, 583-600. 